Pacific Journal of Mathematic 


\section{MULTIPLICATIVE AND EXTREME POSITIVE OPERATORS}

\section{Solveig ESPELIE}

Let $A$ and $B$ denote complex Banach *-algebras and $L(A, B)$ the space of continuous linear operators from $A$ into $B$. Let $P \subset L(A, B)$ be the convex set of positive linear operators of norm $\leqq 1$. If $A$ has an identity, and if $B$ is semi-simple and symmetric, the multiplicative operators of $P$ are shown to be extreme points of $P$. If, on the other hand, it is assumed that, $\|T\|=\|T e\|$ for $T \in P$, then any extreme point $T$ of $P$ satisfies $T e T a b=T a T b$ for all $a, b \in A$. With $A$ as above and $B$ a $B^{*}$-algebra, the extreme points of $P$ are multiplicative. Thus we characterize the extreme points of $P \subset L(A$, $C(X))$ as the multiplicative operators. The results are extended to include the case when $A$ has an approximate identity.

Notation. Let $A$ denote a commutative complex Banach algebra with isometric involution *. We call such an algebra a Banach *-algebra. Let $A^{\prime}$ denote the topological dual space of $A$ and

$$
\hat{P}_{A}=\left\{f \in A^{\prime}: f\left(x x^{*}\right) \geqq 0 \text { for all } x \in A\right\},
$$

the cone of positive functionals on $A$. Define the usual ordering, $\geqq$, on $\hat{P}_{A}$. Further, let

$$
\begin{aligned}
P_{A} & =\left\{f \in \hat{P}_{A}:\|f\| \leqq 1\right\} \\
M_{A}^{\prime} & =\left\{f \in P_{A}: f(x y)=f(x) f(y) \text { for all } x, y \in A\right\} \text { and } \\
M_{A} & =\left\{f \in M_{A}^{\prime}: f \neq 0\right\} .
\end{aligned}
$$

The sets $M_{A}^{\prime}$ and $P_{A}$ are compact in the weak* topology and if $A$ contains an identity (of norm one), then $M_{A}$ is compact in this topology. The set $M_{A}$ is always a weak* closed subset of the maximal ideal space $\Delta_{A}$ of $A$. The set $M_{A}$ is the symmetric portion of $\Delta_{A}$, and if $M_{A}=\Delta_{A}$ we call $A$ a symmetric algebra. Equivalently, $A$ is symmetric if and only if $f\left(x^{*}\right)=\overline{f(x)}$ for all $f \in \Delta_{A}$ and $x \in A$. It is known that the set of extreme points of the convex set $P_{A}$ is exactly $M_{A}^{\prime}$ [4].

We now replace the scalars of the above paragraph by a Banach *-algebra $B$. That is, we consider subsets of $L(A, B)$, the continuous linear operators from $A$ to $B$. An element $T \in L(A, B)$ is called positive if for every $a \in A$ there corresponds a finite set $\left\{b_{i}: i=1, \cdots, n\right\}$ of $B$ such that $T\left(a a^{*}\right)=\sum_{i=1}^{n} b_{i} b_{i}^{*}$. Following the notation of the above paragraph, let $\hat{P}$ be the cone generated by the positive operators and define the usual ordering on $\hat{P}$. Finally, let 


$$
P=\{T \in \hat{P}:\|T\| \leqq 1\}
$$

and

$$
M=\{T \in P: T a b=T a T b \text { for all } a, b \in A\} .
$$

The question examined in this paper is: When is $M$ exactly the set of extreme points of $P$ ? We denote this latter set by ext $P$. With $A$ containing an identity and $B$ semi-simple and symmetric we obtain $M \subset \operatorname{ext} P$. An example exists to show that the semi-simplicity of $B$ is necessary. The symmetry of $B$ appears necessary but we fail to give an example. To obtain the inclusion in the other direction we must require that $B$ be a $B^{*}$-algebra; that is, $\|b\|^{2}=\left\|b b^{*}\right\|$ for all $b \in B$. Equivalently, $B$ is linearly isometric and *-isomorphic to $C\left(\Delta_{B}\right)$ under the map $b \rightarrow \hat{b}$ where $\hat{b}(h)=h(b)$ for all $h \in \Delta_{B}$. By placing a norm condition on $\hat{P}$ we are able to prove the weaker condition that $T e T a b=T a T b$ for $T \in \operatorname{ext} P$ and $a, b \in A$. We say that $\hat{P} \subset L(A, B)$ satisfies Condition I if $\|T\|=\|T e\|$ for all $T \in \hat{P}$.

THE NORM CONDITION. The restriction that $\|T\|=\|T e\|$ for $T \in \hat{P}$ is not unusual. This is a well known property of positive functionals - that is, if $B$ is the set of scalars the condition is satisfied. Further, if $B$ is a $B^{*}$-algebra then we have that $\|T\|=\|T e\|$ when $T \in \hat{P}$. This follows from the fact that in this case the unit ball $S$ of $B^{\prime}$ is the weak* closed absolutely convex hull $\overline{\Gamma\left(\Delta_{B}\right)}$ of $\Delta_{B}$. Hence for $T \in \hat{P}$,

$$
\begin{aligned}
\|T\| & =\sup _{\|b\|=1}\|T b\|=\sup _{\|b\|=1} \sup _{\varphi \in \operatorname{extS}}|\varphi(T b)| \\
& =\sup _{\|b\|=1} \sup _{h \in \Delta_{B}}|\alpha h(T b)|=\sup _{\| \mid D_{1}} \sup _{h \in \Delta_{B}}|h(T b)| \\
& \leqq \sup _{h \in \Delta_{B}}\|h T\|=\sup _{h \in \Delta_{B}} h T e \leqq\|T e\| .
\end{aligned}
$$

Since the reverse inequality is evident it follows that $\|T\|=\|T e\|$. It is conceivable that if Condition I is valid for $\hat{P} \subset L(A, B)$, for every $A$, then $B$ is a $B^{*}$-algebra.

To our knowledge the first work in characterizing the extreme points of such sets as $P$ was done by A. and C. Ionescu Tulcea who considered algebras of real valued continuous functions $C(X)$ and $C(Y)$ on compact Hausdorff spaces $X, Y$. They showed that the extreme points of

$$
P^{\sharp}=\{T \in L(C(X), C(Y)): T \geqq 0 \text { and } T(1)=1\}
$$

are exactly the multiplicative elements of $P^{*}[8,10]$. Various investigators have obtained results related to and extensions of the Ionescu Tulcea result, the work usually being done for algebras of functions (see [2]). In our work the elements of $P$ do not necessarily satisfy $T e=e^{\prime}$. 
In fact when $B$ is a $B^{*}$-algebra and $P^{*}=\left\{T \in \hat{P}\right.$ : Te= $\left.e^{\prime}\right\}$, since $\|T\|=\|T e\|$ for $T \in \hat{P}$, it follows that $P^{\sharp} \subset P$ and moreover, ext $P^{\sharp}=$ $P^{*} \cap \operatorname{ext} P$. Indeed, we obviously have $\left(P^{*} \cap \operatorname{ext} P\right) \subset \operatorname{ext} P^{\sharp}$. Further, if $T \in \operatorname{ext} P^{\sharp}$ and $T \pm S \in P$, then $\|(T \pm S)(e)\|=\left\|e^{\prime} \pm S e\right\| \leqq 1$ and $S e=0$ since $e^{\prime}$ is an extreme point of the unit ball of $B$. But then $(T \pm S)(e)=e^{\prime}$ and $T \pm S \in P^{\sharp}$ so that $S=0$ and $T \in \operatorname{ext} P$. We note that throughout this paper we use the well known characterization of extreme points: If $K$ is a convex set in a linear space $X$ then $x \in \operatorname{ext} K$ if and only if $x \pm y \in K$ for some $y \in X$ implies that $y=0$.

Recently Watanabe in [11] has dropped the requirement that the algebras be commutative and has placed pseudo-norms on the algebras. His results, applied to the commutative case, show that $M \subset \operatorname{ext} P$ when $A$ is $B^{*}$, and the algebra $B$ is semi-simple and symmetric, and both algebras have an identity. Thus, the commutative results in [11] are consequences of our paper since all hypotheses of our theorems are satisfied when $A$ or $B$ are $B^{*}$-algebras with identity.

Theorem 1. Suppose $A$ and $B$ are commutative Banach *algebras, $A$ has an identity, and $B$ is semi-simple and symmetric. If $T \in P$, then $T \in M$ implies $T \in \operatorname{ext} P$.

Proof. Suppose that $T$ is multiplicative and that there exists an element $S \in L(A, B)$ such that $T \pm S \in P$. Let $h \in M_{B}$, then $h T$ is an extreme point of $P_{A}[4]$. Further, $h T \pm h S \geqq 0$ and $\|h T \pm h S\| \leqq 1$. Thus $h S a=0$ for all $a \in A$. But $h$ was arbitrary and $B$ is semi-simple so that $S a=0$ for all $a \in A$, and hence $T \in \operatorname{ext} P$.

The inclusion in the other direction is more difficult to obtain but is valid if we place additional restrictions on the algebras and on the cone of positive operators. We now prove two lemmas which are needed to obtain the implication: If $T$ is an extreme point of $P$ then $T$ is multiplicative.

Lemma 1. Let $A$ be a Banach *algebra and $b$ an element of $A$ such that $b=b^{*}$ and $\|b\|<1$. Then for each $a \in A$, the element a $a^{*}-$ $a b a^{*}$ is of the form $x x^{*}$ for some $x \in A$.

Proof. This is a known result if the algebra $A$ has an identity, for then $e-b=y y^{*}$ where $y=(e-b)^{1 / 2}$ and $y=y^{*}$ (see [6, p. 245]). Hence imbed $A$ in the algebra $A_{1}$ with identity adjoined and write $a a^{*}-a b a^{*}$ as $a(e-b) a^{*}$.

In $A_{1}$ the element $y=(e-b)^{1 / 2}=\sum_{n=1}^{\infty}\left(\begin{array}{c}1 / 2 \\ n\end{array}\right)(-b)^{n}$ exists and in fact, $y=y^{*}$. But then, since $A$ is a maximal ideal in $A_{1}$ and $a \in A$, 
it follows that $x=a y \in A$ and $x x^{*}=(a y)(a y)^{*}=a y^{2} a^{*}=a a^{*}-a b a^{*} \in A$.

Lemma 2. Let $A$ and $B$ be commutative Banach *-algebras and suppose $T \in P$. If $b_{1}, b_{2} \in A$, with $b_{i}=c_{i} c_{i}^{*}$ for some $c_{i} \in A, i=1,2$, define the linear operator $S$ by

$$
S(a)=T\left(b_{1}\right) T\left(a b_{2}\right)-T\left(b_{2}\right) T\left(a b_{1}\right) \text { for } a \in A .
$$

Then $T \pm S \geqq 0$.

Proof. For any $a \in A$,

$$
\begin{aligned}
(T+S)\left(a a^{*}\right) & =T\left(a a^{*}\right)+T\left(b_{1}\right) T\left(b_{2} a a^{*}\right)-T\left(b_{2}\right) T\left(b_{1} a a^{*}\right) \\
& \geqq T\left(a a^{*}\right)-T\left(b_{2}\right) T\left(b_{1} a a^{*}\right) \\
& =T\left(p_{1}\right)+T\left(b_{1} a a^{*}\right)-T\left(b_{2}\right) T\left(b_{1} a a^{*}\right) \\
& \geqq T\left(b_{1} a a^{*}\right)-T\left(b_{2}\right) T\left(b_{1} a a^{*}\right) \geqq 0 .
\end{aligned}
$$

Since $\left\|b_{1}\right\|<1$ and $b_{1}=c_{1} c_{1}^{*}$ from Lemma 1 it follows that the element $p_{1}=a a^{*}-b_{1} a a^{*}$ is positive. Similarly, since $\left\|T\left(b_{2}\right)\right\|<1$ and $T\left(b_{2}\right)$ is self-adjoint, and since $T\left(b_{1} a a^{*}\right)$ is positive, with repeated applications of Lemma 1 it follows that $T\left(b_{1} a a^{*}\right)-T\left(b_{2}\right) T\left(b_{1} a a^{*}\right)$ is of the form $\sum_{i=1}^{n} c_{i} c_{i}{ }^{*}$ in $B$. In a similar way it can be shown that ( $T-$ $S)\left(a a^{*}\right) \geqq 0$ for $a \in A$.

Theorem 2. Suppose that $A$ and $B$ are commutative Banach *-algebras, that $A$ has an identity and that Condition I holds for elements of $\hat{P}$. Then $T e T a b=T a T b$ for all $a, b \in A$ whenever $T$ is an extreme point of $P$.

Proof. Suppose that $T \in \operatorname{ext} P$ and $b \in A$, with $b=c c^{*}$ for some $c \in A$ and $\|b\|<1$. We let $S(a)=1 / 2(T(b) T(a)-T(e) T(a b))$ be the operator defined in Lemma 2 (taking $b_{1}=b$ and $b_{2}=1 / 2 e$ ), so that $T \pm S \geqq 0$.

Since $\|T \pm S\|=\|(T \pm S)(e)\|=\|T e\| \leqq 1$ and $T \in \operatorname{ext} P$ it follows that $T e T a b=T a T b$ for all $a \in A$ provided that $b=c c^{*}$ and $\|b\|<1$. But every element $b \in A$ is a linear combination of at most four elements of the form $c c^{*}$ with $\left\|c c^{*}\right\|<1$ and hence $T e T a b=T a T b$ for all $a, b \in A$.

COROLLARY 2.1. With the hypotheses of Theorem 2 it follows that $h T$ lies on an extreme ray of $\hat{P}_{A}$ for every $h \in M_{B}$ and $T \in \operatorname{ext} P$.

Proof. Since $T e T a b=T a T b$ for all $a, b \in A$ and $\|h T\|=h T e$ for all $h \in M_{B}$ it follows that if $\|h T\| \neq 0$ then $(h T /\|h T\|)(a b)=$ 
$(h T /\|h T\|)(a)(h T /\|h T\|)(b)$ for $a, b \in A$ and $(h T /\|h T\|) \in M_{A}$. But then, either $\|h T\|=0$ or $h T /\|h T\| \in \operatorname{ext} P_{A}$; thus $h T$ lies on an extreme ray of $\hat{P}_{A}$ for each $h \in M_{B}$.

COROLlaRY 2.2. Suppose that the hypotheses of Theorem 2 hold and in addition, that $B$ is semi-simple and symmetric. Then $T$ is multiplicative if $\|h T\|$ is 0 or 1 for each $h \in M_{B}$ and $T \in \operatorname{ext} P$.

Proof. Since $h T$ lies on an extreme ray of $\hat{P}_{A}$ and $\|h T\|$ is 0 or 1 then $h T \in M_{A}$ for each $h \in M_{B}$. But then, $h(T a b)=h(T a T b)$ for all $a, b \in A$ and $h \in M_{B}$, and since $B$ is semi-simple and symmetric, it follows that $T$ is multiplicative.

THEOREM 3. Let $A$ be a commutative Banach *algebra with identity and let $B$ be a $B^{*}$-algebra. Then every extreme point $T$ of $P$ is multiplicative.

Proof. If $T \in \operatorname{ext} P$ it follows from Theorem 2 that $T e T a b=$ $T a T b$ for all $a, b \in A$. Thus it suffices to prove that $T e T a=T a$ for all $a \in A$.

Let $S(a)=1 / 2(T e T a-T a)$ for $a \in A$. The method of proof of Lemma 2 shows that $T \pm S \geqq 0$ and so we need only prove that $\| T \pm$ $S \| \leqq 1$. Since $B$ is a $B^{*}$-algebra and $T \pm S \geqq 0$ it follows from our earlier remarks that $\|T \pm S\|=\|(T \pm S)(e)\|$. Now, $\|T+S\|=$ $\|(T+S)(e)\|=\left\|1 / 2 T e+1 / 2(T e)^{2}\right\| \leqq 1 / 2\|T e\|+1 / 2\|T e\|^{2} \leqq 1$. Furthermore, since $0 \leqq T e-S e=(3 / 2) T e-1 / 2(T e)^{2}$ and $\|T e\| \leqq 1$ we see (letting $f=\widehat{T e} \in C\left(\Delta_{B}\right)$ ) that $f \geqq 0$ and $\|f\| \leqq 1$. But then $0 \leqq f \leqq 1$ so that $(1-f)(2-f) \geqq 0$ and therefore $0 \leqq(3 / 2) f-1 / 2 f^{2} \leqq 1$. Hence $\|T e-S e\|=\left\|(3 / 2) f-1 / 2 f^{2}\right\| \leqq 1$ and the proof is complete.

Theorem 4. Assume that $A, B$ are commutative Banach *algebras, that $A$ has an identity and that $B$ is semi-simple and symmetric. Let $T \in L(A, B)$.

(a) If $T \in \operatorname{ext} P$ and $h T$ lies on an extreme ray of $\hat{P}_{A}$ for all $h \epsilon$ $M_{B}$, then $T e T a b=T a T b$ for $a, b \in A$.

(b) If $T \in \operatorname{ext} P$ and $h T=0$ or $h T \in \operatorname{ext} P_{A}$ for all $h \in M_{B}$, then $T a b=T a T b$ for all $a, b \in A$.

The proofs of (a) and (b) are immediate for if $h T$ lies on an extreme ray of $\hat{P}_{A}$, then either $\|h T\|=0$ or $h T /\|h T\| \in M_{A}$ for each $h \in M_{B}$. But then $h(T e T a b)=h(T a T b)$ for all $h \in M_{B}$, and since $B$ is semi-simple and symmetric, it follows that $T e T a b=T a T b$ for $a, b \in$ $A$. Finally, if $h T e$ is 0 or 1 for all $h \in M_{B}$, then $T$ is multiplicative. We state the following result without proof. Using the above 
methods and Theorem 3 the result follows.

THEOREM 5. Let $A$ be a commutative Banach *-algebra with identity and $B$ a $B^{*}$-algebra. If $T \in P$ and $T e T a b=T a T b$ for $a, b \in$ $A$, then $T \in \operatorname{ext} P$ if and only if $T e$ is an extreme point of $K=\{b \in$ $B:\|b\| \leqq 1$ and $b=\sum_{i=1}^{n} c_{i} c_{i}^{*}, n$ finite $\}$.

It should be noted that if $T e \in \operatorname{ext} K$ and $T e T a b=T a T b$ for $a$, $b \in A$ then $T \in \operatorname{ext} P$ when $B$ is semi-simple and symmetric. To obtain the converse we employ the hypothesis that $B$ is a $B^{*}$-algebra so that $K$ can be identified with the set $\{f: f \geqq 0$ and $\|f\| \leqq 1\}$ in $C\left(\Delta_{B}\right)$. It is well known that the set of extreme points of this set is $\{f: f(x)=$ 0 or 1 for all $\left.x \in \Delta_{B}\right\}$.

ExAmples. We now display some examples indicating the need for the hypotheses placed on the algebras $A$ and $B$ in the above work. Most of the algebras used in our examples can be found in [10].

Consider the involution algebra $\mathscr{A}$ of functions analytic on the open unit disc and continuous on the closed disc with the usual supremum norm and pointwise multiplication. An involution is defined on $\mathscr{A}$ by $f^{*}(z)=\overline{f(z)}$. The algebra $\mathscr{A}$ is semi-simple and not symmetric.

We construct an element of ext $P \subset L(\mathscr{A}, \mathscr{A})$ which is not in $M$. Denote by $h$ the element of $P$ defined by $h(f)=f(1)$ for $f \in \mathscr{A}$ and denote by $Z^{n}$ the element of $\mathscr{A}$ defined by $Z^{n}(w)=w^{n}$ for $n=0,1$, $2, \cdots$. Then the operator given by $T f=Z^{2} h(f)$ for $f \in \mathscr{A}$ is an element of ext $P$ and not in $M$.

It follows that $T \in P$ since $T\left(f f^{*}\right)=f(1) f^{*}(1) Z^{2}=(f(1) Z)(f(1) Z)^{*}$ and $\|T\|=\sup _{\|f\|=1}\left\|Z^{2} h(f)\right\|=1$. Suppose that $T \pm S \in P$ for some $S \in L(\mathscr{A}, \mathscr{A})$ so that $\left\|T\left(Z^{n}\right) \pm S\left(Z^{n}\right)\right\|=\left\|Z^{n} \pm S\left(Z^{n}\right)\right\| \leqq 1$. Since $Z^{n}$, for each $n$, is an extreme point of the unit ball of $\mathscr{A}$ it follows that $S\left(Z^{n}\right)=0$ and that $S$ is zero on the polynomials, a dense subalgebra of $\mathscr{A}$. Consequently, $S=0$ and $T \in \operatorname{ext} P . T$ is not multiplicative but it should be noted that $T$ satisfies $T e T f g=T f T g$ for all $f$, $g \in \mathscr{A}$.

We now consider a space $L(A, B)$ such that the algebra $B$ is not semi-simple. Let $\mathscr{A}$ be the Banach space of the above paragraph. However, we now place a multiplication on $\mathscr{A}$ defined in terms of a convolution, $f * g(w)=w \int_{0}^{1} f[(1-t) w] g(t w) d t$ where $|w| \leqq 1$. We denote this algebra by $\mathscr{A}_{0}$; it is a Banach *-algebra with the involution $f^{*}(z)=\overline{f(z)}$ and the supremum norm. With this definition of multiplication we have $\lim _{n \rightarrow \infty}\left\|f^{n}\right\|^{1 / n}=0$ for all $f \in \mathscr{A}_{0}$ since $\left\|f^{n}\right\|=$ $\|f\|^{n} /(n-1)$ !. Thus $\mathscr{A}_{0}$ is a radical algebra and we consider the 
algebra $\mathscr{A}_{0}+e$, the algebra with the identity adjoined. This algebra has one maximal ideal so that it is symmetric. The element $Z(w)=$ $w$ is positive since it is $I^{2}$ where $I(w)=1$ for all $w$ in the disc.

We consider $L\left(\mathscr{A}, \mathscr{A}_{0}+e\right)$ and define $T \in L\left(\mathscr{A}, \mathscr{A}_{0}+e\right)$ by $T f=$ $f(1) Z, Z(w)=w$. Using methods similar to these above we can show that $T \in \operatorname{ext} P$ and $T \notin M$. All the hypotheses of Theorem 4.b are satisfied except for the semi-simplicity of the range space. Again we have $T e T f g=T f T g$ for all $f, g \in \mathscr{A}$.

Finally we display an operator between two algebras which is multiplicative and not an extreme point of $P$ when all hypotheses of Theorem 1 are satisfied except for the semi-simplicity of the range.

Let $\Omega$ be the algebra of all power series $a(z)=\sum_{n=0}^{\infty} a_{n} z^{n}$ such that $\sum_{n=0}^{\infty}\left|a_{n}\right| / n !<\infty$; the norm is $\|a(z)\|=\sum_{n=0}^{\infty}\left(\left|a_{n}\right| / n !\right)$. Multiplication is defined in the usual way. $\Omega$ is a Banach *algebra with involution defined by $(a(z))^{*}=\sum_{n=0}^{\infty} \bar{a}_{n} z^{n}$. The identity of $\Omega$ is the series with $a_{0}=1$ and $a_{n}=0$ for $n \geqq 1$.

We consider the maximal ideal generated by the series with $a_{1}=$ 1 and $a_{n}=0$ for $n \neq 1$. All elements of this ideal are essentially nilpotent and this is the only maximal ideal in $\Omega$. Thus $\Omega$ is symmetric and not semi-simple. Let $T \in L(\Omega, \Omega)$ be defined by $T\left(\sum_{n=0}^{\infty} a_{n} z^{n}\right)=\sum_{n=0}^{\infty} a_{n} z^{2 n}$. It can be shown that $T \in P \subset L(\Omega, \Omega)$ and that $T \in M$. Define $S$ by $S(a(z))=\sum_{n=1}^{\infty} a_{2 n-1} z^{4 n-2}$. Now $T \pm S \in P$ and $S \neq 0$ so that $T \notin \operatorname{ext} P$.

A Generalization. If we replace the condition that $A$ has an identity with the condition that $A$ has an approximate identity we obtain analogues of the above statements. The net $\left\{e_{\alpha}\right\}$ is an approximate identity in $A$ if $\left\|e_{\alpha}\right\| \leqq 1$ and $e_{\alpha}>0$ for all $\alpha$ and $\left\|e_{\alpha} x-x\right\| \longrightarrow{ }_{\alpha}$ 0 for all $x \in A$. (We assume that $e_{\alpha}>0$ for all $\alpha$ since $\left\|x e_{\alpha} e_{\alpha}^{*}-x\right\|^{\alpha} \leqq$ $\left\|\left(x e_{\alpha}-x\right) e_{\alpha}^{*}\right\|+\left\|x e_{\alpha}^{*}-x\right\| \leqq\left\|x e_{\alpha}-x\right\|+\left\|x^{*} e_{\alpha}-x^{*}\right\|$ for all $x \in A$ so that $\left\{e_{\alpha} e_{\alpha}^{*}\right\}$ is an approximate identity whenever $\left\{e_{\alpha}\right\}$ is.) We make use of the fact that for a commutative Banach *-algebra $A$ with approximate identity, if $f \in \widehat{P}_{A}$, then $\|f\|=\lim _{\alpha} f\left(e_{\alpha}\right)$ and $M_{d}^{\prime}=\operatorname{ext} P_{A}$ [5]. With this result Theorem 1 remains true as stated in this new setting.

To obtain further generalizations we place Condition I' on $\hat{P}:\|T\|=$ $\lim _{\alpha}\left\|T e_{\alpha}\right\|$ for all $T \in \hat{P}$. We now show that if $T$ is positive in $L(A, B)$ where $A$ is a commutative Banach *-algebra with approximate identity and $B$ is a $B^{*}$-algebra, then Condition $I^{\prime}$ holds. For each $\alpha$ and each $h \in M_{B}$ we have $h T e_{\alpha} \leqq\left\|T e_{\alpha}\right\|$ so that $\|h T\|=\lim _{\alpha} h T e_{\alpha}=$ $\lim \inf _{\alpha} h T e_{\alpha} \leqq \liminf f_{\alpha}\left\|T e_{\alpha}\right\|$. Since $\|T\| \leqq \sup _{\|h\|=1}\|h T\|$, it follows that $\|T\| \leqq \liminf f_{\alpha}\left\|T e_{\alpha}\right\|$. Moreover, $\left\|T e_{\alpha}\right\| \leqq\|T\|$ for each $\alpha$ yielding $\lim \sup _{\alpha}\left\|T e_{\alpha}\right\| \leqq\|T\|$ and hence $\|T\|=\lim _{\alpha}\left\|T e_{\alpha}\right\|$.

THeorem 6. Suppose that $A, B$ are commutative Banach *algebras, 
that $A$ has an approximate identity, and that $\hat{P}$ satisfies Condition I'. Then, for every extreme point $T$ of $P$ it follows that

$$
T(a) T(b)=\lim _{\alpha} T\left(e_{\alpha}\right) T(a b) \text { for all } a, b \in A .
$$

Proof. Let $S(a)=1 / 2\left(T(b) T\left(e_{\alpha} a\right)-T\left(e_{\alpha}\right) T(b a)\right)$ be the operator defined in Lemma 2 with $b_{1}=b$ and $b_{2}=e_{\alpha} / 2$, so that $T \pm S \geqq 0$.

Since $\hat{P}$ satisfies Condition $I^{\prime}$, it follows that $\|T \pm S\|=\lim _{\beta} \| T\left(e_{\beta}\right) \pm$ $1 / 2\left[T(\mathrm{~b}) T\left(e_{\alpha} e_{\beta}\right)-T\left(e_{\alpha}\right) T\left(b e_{\beta}\right)\right]\left\|=\lim _{\beta}\right\| T\left(e_{\beta}\right) \| \leqq 1$ and hence, $T \pm S \in P$. Since $T \in \operatorname{ext} P$ we have $T(b) T\left(e_{\alpha} a\right)=T\left(e_{\alpha}\right) T(b a)$ for $a \in A$ and each $e_{\alpha}$, so that $\lim _{\alpha} T\left(e_{\alpha}\right) T(a b)=T(a) T(b)$ for all $a \in A$ and $b \in A$ with $\|b\|<1$ and $b=c c^{*}$ for some $c \in A$.

Now, every product, and hence every element of the form $b e_{\beta}$ can be written as the linear combination of four positive elements; that is, $b e_{\beta}=1 / 4 \sum_{k=1}^{4} \bar{i}^{k}\left(e_{\beta}^{*}+i^{k} b\right)\left(e_{\beta}^{*}+i^{k} b\right)^{*}$ for all $b \in A$ and all $\beta$. It follows, from the linearity of $T$, that $\lim _{\alpha} T\left(e_{\alpha}\right) T\left(a b e_{\beta}\right)=T(a) T\left(b e_{\beta}\right)$ for all $a, b \in A$ and all $\beta$; and, from the continuity of $T$, that $\lim _{\alpha} T\left(e_{\alpha}\right) T(a b)=T(a) T(b)$ for $a, b \in A$.

CoROLlaRY 6.1. With the hypotheses of Theorem 6 for $T \in \hat{P}$ and $h \in M_{B}$, either $h T=0$ or $h T /\|h T\| \in M_{A}$.

Proof. From Theorem 6 for $T \in \hat{P}$ we conclude that $\lim _{\alpha} h\left(T e_{\alpha} T a b\right)=$ $h(T a T b)$ for $a, b \in A$ and $h \in M_{B}$. Consequently,

$$
\|h T\| h T(a b)=h T(a) h T(b)
$$

and either $h T=0$ or $h T /\|h T\| \in M_{A}$ for $h \in M_{B}$. Or equivalently, $h T$ lies on an extreme ray of $\hat{P}_{A}$ for every $h \in M_{B}$ and $T \in \hat{P}$.

Definition. As noted earlier, if $A$ is a commutative Banach *-algebra then $A_{1}$ denotes the algebra with identity adjoined. If $f \in$ $\hat{P}_{A}$ then $f_{1}$, defined by $f_{1}(a+\lambda)=f(a)+\lambda\|f\|$, is the extension of $f$ to $A_{1}$ and $\hat{P}_{A_{1}}$ is the cone of positive elements of $A_{1}^{\prime}$. Similarly, $P_{A_{1}}$, denotes the set of positive elements of norm $\leqq 1$. Finally, if $T \in L(A, B)$ and $T \geqq 0$ we define $T_{1}$ to be that element of $L\left(A_{1}, B_{1}\right)$ defined by $T_{1}(a+\lambda)=T(a)+\lambda\|T\|$. (We let $B_{1}=B$ if $B$ has an identity.) Furthermore, $\hat{P}_{1}$ denotes the cone of positive operators and $P_{1}$ those positive operators of norm $\leqq 1$.

We now prove the statement equivalent to Theorem 3 when $A$ has an approximate identity.

THEOREM 7. Let $A$ be a commutative Banach *-algebra with approximate identity and $B$ a $B^{*}$-algebra. Then, every extreme point $T$ of $P$ is multiplicative. 
Proof. From the decomposition for products used in Theorem 6 it follows easily that if $T$ is a positive operator, then $T\left(a b^{*}\right)=\left(T\left(a^{*} b\right)\right)^{*}$ for $a, b \in A$. Since $A$ has an approximate identity and $T$ is continuous, it follows that $T\left(a^{*}\right)=(T a)^{*}$ for $a \in A$. Moreover, since the range of $T$ is contained in $C_{0}\left(\Delta_{B}\right)$ (or $C\left(\Delta_{B}\right)$ if $B$ contains an identity), letting $h_{x}$ (or $x$ ) be that element of $\Delta_{B}$ defined by $h_{x}(b)=b(x)$ for $b \in C_{0}\left(\Delta_{B}\right)$ it follows that $\left(T a^{*}\right)(x)=\overline{(T a)(x)}$ for $a \in A$ and $x \in \Delta_{B}$. Further, for $T$ positive from a Cauchy-Schwarz inequality [9, p. 213] we conclude that $\left|T\left(a b^{*}\right)\right|^{2}(x) \leqq\left[T\left(a a^{*}\right) T\left(b b^{*}\right)\right](x)$ for $a, b \in A$ and $x \in \Delta_{B}$. Letting $b=e_{\alpha}$ and taking the limit we obtain $|T a|^{2}(x)=\|T\|\left[T\left(a a^{*}\right)\right](x)$ for $x \in \Delta_{B}$ and $a \in A$.

We now show that for any $T \in \hat{P}$ the element $T_{1}$ defined by $T_{1}(a+$ $\lambda)=T a+\lambda\|T\|$ is, in fact, positive. Thus, if $T \in \hat{P}$ and $\|T\| \neq 0$, then

$$
\begin{aligned}
{\left[T_{1}(a+\lambda)\left(a^{*}+\bar{\lambda}\right)\right](x) } & =\left[T a a^{*}+\lambda T a^{*}+\bar{\lambda} T a+|\lambda|^{2}\|T\|\right]\left(x_{1}\right) \\
& =\left[T a a^{*}-2 R e(-\bar{\lambda}) T a+|\lambda|^{2}\|T\|\right]\left(x_{1}\right) \\
& \geqq\left[1 /\|T\||T a|^{2}-2|\lambda||T a|+|\lambda|^{2}\|T\|\right]\left(x_{1}\right) \\
& =1 /\|T\|[|T a|-\|T\||\lambda|]^{2}\left(x_{1}\right) \geqq 0
\end{aligned}
$$

for all $a+\lambda \in A_{1}$ and $x_{1} \in \Delta_{B_{1}}$, where $x_{1}$ is the extension of the element $x$ in $A_{B}$ when $B$ does not have an identity. Finally, when $B$ does not have an identity, let $x^{\prime}$ be that element of $\Delta_{B_{1}}$ which has $A$ as its corresponding maximal ideal (we note that in this case $\Delta_{B_{1}}$ is the one point compactification of $\left.\Delta_{B}\right)$; then $\left[T_{1}(a+\lambda)\left(a^{*}+\bar{\lambda}\right)\right]\left(x^{\prime}\right)=|\lambda|^{2}\left(x^{\prime}\right) \geqq 0$ for $a+\lambda \in A_{1}$. Thus $T_{1} \geqq 0$. Since the range of $T_{1}$ is contained in a $B^{*}$-algebra, $\hat{P}_{1}$ satisfies Condition $I$ and hence $\left\|T_{1}\right\|=\left\|T_{1} e\right\|$.

We now show that $T_{1}$ is an extreme point of $P_{1}$. Suppose that there exists $S \in L\left(A_{1}, B_{1}\right)$ such that $T_{1} \pm S \in P_{1}$. Then, $\left\|T_{1} e \pm S e\right\|=$ $\left\|e^{\prime} \pm S e\right\| \leqq 1$ and hence $S e=0$ since $e^{\prime}$ is an extreme point of the unit ball of $B_{1}$. Let $\bar{S}$ denote the restriction of $S$ to $A$, then, since $T \pm \bar{S} \geqq 0$ and $\|T \pm \bar{S}\| \leqq 1$, it follows that $\bar{S}=0$ since $T \in \operatorname{ext} P$. Therefore, $S=0$ and $T_{1}$ is an extreme point of $P_{1}$ so that from Theorem 3 it follows that $T_{1}$ is multiplicative. Hence, $T$ is multiplicative and the proof is complete.

Acknowledgements. I wish to express appreciation to George Maltese for his many helpful conversations and to R. R. Phelps for bringing many improvements in the paper to my attention.

\section{REFERENCES}

1. R. M. Blumenthal, Joram Lindenstrauss, and R. R. Phelps, Extreme Operators into $C(K)$, Pacific J. Math., 15 (1965), 747-756.

2. F. F. Bonsall, J. Lindenstrauss, and R. R. Phelps, Extreme Positive Operators on Algebras of Functions, Math. Scand., 18 (1966), 161-182. 
3. N. Bourbaki, Espaces Vectoriels Topologiques, Chaps. I-II, 2nd Ed., Hermann, Paris, 1966.

4. R. S. Bucy and G. Maltese, A representation theorem for positive functionals on involution algebras, Math. Annalen, 162 (1966), 364-367.

5. M. S. Espelie, Extreme Functionals, (submitted).

6. I. Gelfand, D. Raikov, and G. Shilov, Commutative Normed Rings, Chelsea, New York, 1964.

7. L. H. Loomis, An Introduction to Abstract Harmonic Analysis, D. Van Nostrand Co., Princeton, 1953.

8. R. R. Phelps, Extreme positive operators and homomorphisms, Trans. Amer. Math. Soc., 108 (1963), 265-274.

9. C. E. Rickart, General Theory of Banach Algebras, D. Van Nostrand Co., Princeton, 1960 .

10. A. and C. Ionescu Tulcea, $A$ note on extremal points, (unpublished).

11. S. Watanabe, Positive linear maps of Banach algebras with an involution, Sci. Rep. Niigata Univ. Ser. A. Nov., 8 (1971), 1-6.

Received August 31, 1971 and in revised form April 18, 1973.

HOWARD UNIVERSITY 


\section{PACIFIC JOURNAL OF MATHEMATICS}

\section{EDITORS}

RICHARD ARENS (Managing Editor)

University of California

Los Angeles, California 90024

R. A. Beaumont

University of Washington

Seattle, Washington 98105
J. DUGUNDJI*

Department of Mathematics

University of Southern California

Los Angeles, California 90007

D. Gilbarg and J. Milgram

Stanford University

Stanford, California 94305

\section{ASSOCIATE EDITORS}
E. F. BECKENBACH
B. H. NeumanN
F. WOLF
K. YosHIDA

\section{SUPPORTING INSTITUTIONS}

UNIVERSITY OF BRITISH COLUMBIA
CALIFORNIA INSTITUTE OF TECHNOLOGY
UNIVERSITY OF CALIFORNIA
MONTANA STATE UNIVERSITY
UNIVERSITY OF NEVADA
NEW MEXICO STATE UNIVERSITY
OREGON STATE UNIVERSITY
UNIVERSITY OF OREGON
OSAKA UNIVERSITY

UNIVERSITY OF BRITISH COLUMBIA

UNIVERSITY OF CALIFORNIA

MONTANA STATE UNIVERSITY

UNIVERSITY OF NEVADA

OREGON STATE UNIVERSITY

OSAKA UNIVERSITY
UNIVERSITY OF SOUTHERN CALIFORNIA

STANFORD UNIVERSITY

UNIVERSITY OF TOKYO

UNIVERSITY OF UTAH

WASHINGTON STATE UNIVERSITY

UNIVERSITY OF WASHINGTON

\section{AMERICAN MATHEMATICAL SOCIETY} NAVAL WEAPONS CENTER

* C. R. DePrima California Institute of Technology, Pasadena, CA 91109, will replace J. Dugundji until August 1974. 


\section{Pacific Journal of Mathematics}

\section{Vol. 48, No. $1 \quad$ March, 1973}

Jan Aarts and David John Lutzer, Pseudo-completeness and the product of Baire

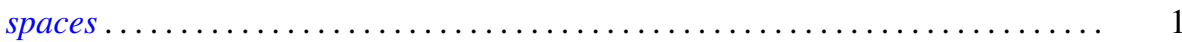

Gordon Owen Berg, Metric characterizations of Euclidean spaces ............ 11

Ajit Kaur Chilana, The space of bounded sequences with the mixed topology ..... . 29

Philip Throop Church and James Timourian, Differentiable open maps of

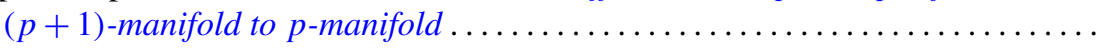

P. D. T. A. Elliott, On additive functions whose limiting distributions possess a finite

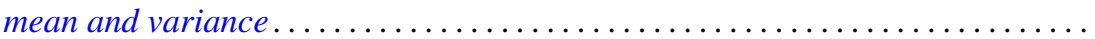

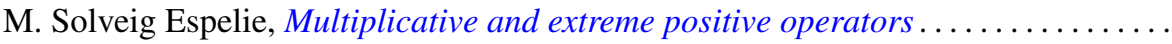

Jacques A. Ferland, Domains of negativity and application to generalized convexity

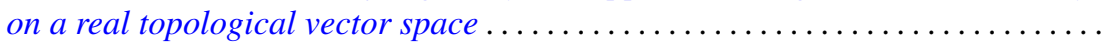

Michael Benton Freeman and Reese Harvey, A compact set that is locally holomorphically convex but not holomorphically convex ...............

Roe William Goodman, Positive-definite distributions and intertwining

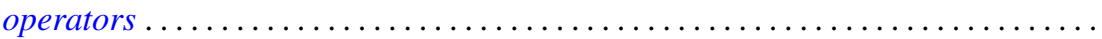

Elliot Charles Gootman, The type of some $C^{*}$ and $W^{*}$-algebras associated with

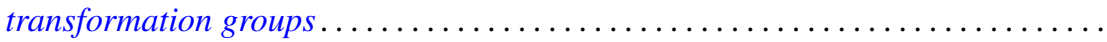

David Charles Haddad, Angular limits of locally finitely valent holomorphic

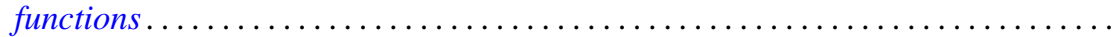

William Buhmann Johnson, On quasi-complements .

William M. Kantor, On 2-transitive collineation groups of finite projective spaces...

Joachim Lambek and Gerhard O. Michler, Completions and classical localizations of right Noetherian rings

Kenneth Lamar Lange, Borel sets of probability measures ......

David Lowell Lovelady, Product integrals for an ordinary differential equation in a Banach space

Jorge Martinez, A hom-functor for lattice-ordered groups .........

W. K. Mason, Weakly almost periodic homeomorphisms of the two sphere ....

Anthony G. Mucci, Limits for martingale-like sequences .......

Eugene Michael Norris, Relationally induced semigroups ...

Arthur E. Olson, A comparison of c-density and $k$-density ......

Donald Steven Passman, On the semisimplicity of group rings of linear groups.

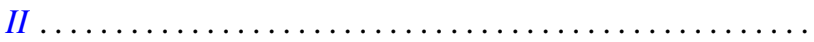

Charles Radin, Ergodicity in von Neumann algebras .

P. Rosenthal, On the singularities of the function generated by the Bergman operator of the second kind.

Arthur Argyle Sagle and J. R. Schumi, Multiplications on homogeneous spaces,

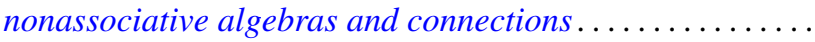

Leo Sario and Cecilia Wang, Existence of Dirichlet finite biharmonic functions on

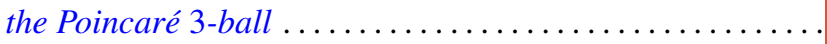

Ramachandran Subramanian, On a generalization of martingales due to Blake ..

Bui An Ton, On strongly nonlinear elliptic variational inequalities.

Seth Warner, A topological characterization of complete, discretely valued

fields. 